responds to $S_{0} \rightarrow S_{1}\left(\pi^{*}\right.$ No $\rightarrow\left(\sigma^{*}\right.$ No, $\left.3 S_{N}\right)$ ) of Table IV.

The 3-alkyl derivatives of 4 differ from the parent 4 and its $\mathrm{N}$-alkyl derivative 2-Me-4 in that the quasi-degeneracy of the $\mathrm{S}_{0}$ $\rightarrow S_{2}$ and $S_{0} \rightarrow S_{3}$ transitions is lifted. The first three transitions of $(2 R, 3 R)-3-\mathrm{Me}-4$ are all calculated to have negative rotatory strengths, the third transition, $\pi^{*}$ NO $\rightarrow 3 p$, displaying appreciable intensity. The cis isomer $(2 S, 3 R)-3-\mathrm{Me}-4$ is the only oxaziridine investigated which has the $S$ absolute configuration at $\mathrm{N}$ and whose first transition has a negative $C D$. The intensity is weak, however. The CD of the second transition is positive and is of greater magnitude. In none of the species with the $R$ configuration at $\mathrm{N}$ is there a transition calculated to occur below $10 \mathrm{eV}$ which appears to have a strong enough positive rotatory strength to outweigh the negative $C D$ of the lower transitions.

\section{Conclusions}

The lower transitions of all of the oxiranes, aziridines, diaziridines, and oxaziridines of this study originate from nonbonded electron pairs and terminate in diffuse orbitals which have appreciable mixed-valence Rydberg character. A disagreement exists between the calculated and measured sign of the CD of the first transition of $(-)-(S)-2-\mathrm{Me}-1$. The discrepancy is probably due to the level of CI used in this study which is not able to describe substantial reorganization of the "unexcited" electrons. In the oxirane system, the only one of the present systems where such reorganization is important as suggested by the magnitude of the Koopmans' defect, the consequent inadequate description of the lower excited states apparently results in the wrong computed sign of the CD of the first (and possibly other) transitions.

The first transition of $(-)-(1 S, 2 S)-2$-methylaziridine has a positive rotational strength. The signs of the $C D$ of all of the lower electronic transitions of this compound with the possible exception of the second are determined by the configuration at $\mathrm{N}$ rather than at $C$.

The first two transitions of $(R, R)$-diaziridine and $(R, R)-1$ methyl- and $(R, R)$-3-methyldiaziridine both have negative $C D$. In the chiral diaziridines where the absolute configuration at the $\mathrm{N}$ atoms has been experimentally determined, compounds with the $(R, R)$ configurations at the $\mathrm{N}$ atoms are, without exception, dextrorotatory at longer wavelengths. At least in the case of the mono- $N$-alkyldiaziridines, the observed sign of rotation at longer wavelengths may be due to the Cotton effect of the fourth transition, identified as $\pi^{*} \mathrm{~N} \rightarrow 3 \mathrm{~s}$.

The first two transitions of $(R)$-oxaziridine and $(R)-N$ methyloxaziridine have negative Cotton effects, as does the $(2 R, 3 R)$-3-methyloxaziridine. The first transition of $(2 S, 2 R)$ 3-methyloxaziridine also has a negative $C D$, indicating that optical activity of oxaziridines is not dominated by the configuration at N.

Acknowledgment. This work was supported by the Natural Sciences and Engineering Research Council of Canada and by the award of a Killam Resident Fellowship by the University of Calgary. Generous grants of computing time by the University of Calgary and the Northern Alberta Institute of Technology are gratefully acknowledged.

\title{
High-Resolution Optical Studies on C-Phycocyanin via Photochemical Hole Burning
}

\author{
J. Friedrich, ${ }^{\dagger}$ H. Scheer, ${ }^{\ddagger}$ B. Zickendraht-Wendelstadt, ${ }^{\S}$ and D. Haarer* \\ Contribution from IBM Research Laboratory, San Jose, California 95193. \\ Received August 17, 1980
}

\begin{abstract}
We have shown that both the native $C$-phycocyanin and its corresponding free biline chromophore undergo reversible, low-temperature photochemistry. We attribute this photochemistry to reversible proton-transfer processes and utilize the observed photoreaction for photochemical hole burning (PHB). Using narrow-band PHB experiments, we have been able to perform high-resolution optical studies and show that the protein-chromophore assembly forms a very rigid structure. The results lead to the conclusion that the light-induced proton transfer occurs most probably in the triplet state.
\end{abstract}

\section{Introduction}

$C$-Phycocyanins (C-PC) are known to be the light-harvesting pigments of blue green and red algae. ${ }^{1-4}$ The molecules contain the bile pigment chromophores, i.e., absorbers with high oscillator strength, which are covalently bound to large proteins. $C$-PC is composed of two protein subunits with molecular weights in the range of $2 \times 10^{4}$. It contains three chromophores, one in the $\alpha$ and two in the $\beta$ subunit.

Conformational studies on $C$-phycocyanin have been performed with optical absorption spectroscopy. ${ }^{5}$ These studies can be summarized as follows:

(a) The protein environment stabilizes certain chromophore configurations and thus reduces the optical line width of the bile pigment chromophores, which can (in their unstabilized forms) attain various configurations varying from cyclic porphyrin-type

\footnotetext{
IBM Postdoctoral Fellow. Permanent address: Institut fuer Physikalische und Theoretische Chemie, TU Muenchen, West Germany.

Institut fuer Botanik, Universitaet Muenchen, West Germany.

Institut fuer Physikalische und Theoretische Chemie, TU Muenchen, West Germany.
}

structures to elongated polyene-type structures. The coexistence of these configurations leads to a relatively large inhomogeneous bandwidth of the optical spectra of free bile pigments in the solid state or in liquid solution. 5

(b) The most likely chromophore structure of native $C$-PC is an elongated polyene-type conformation which is characterized by a very strong optical absorption in the low-energy band $(6000$ $\AA)$ and by a weaker absorption in the near-UV region (3500 A). ${ }^{5,7-9}$

(1) A. N. Glazer, Photochem. Photobiol. Rev., 71 (1976).

(2) P. O'Carra, C. O'hEocha, and T. W. Goodwin, "Chemistry and Biochemistry of Plant Pigments", Academic Press, New York, 1976.

(3) H. Scheer, Angew. Chem., in press.

(4) A. N. Glazer, Mol. Cell. Biochem., 18, 125 (1977).

(5) H. Scheer and W. Kufer, Z. Naturforsch., C, 32c, 513 (1977).

(6) A. R. Holzwarth, H. Lehner, S. E. Braslavsky, and K. Schaffner, Liebigs Ann. Chem., 2002 (1978).

(7) M. J. Burke, D. C. Pratt, and A. Moscowitz, Biochemistry, 11, 4025 (1972)

(8) Q. Chae and P. S. Song, J. Am. Chem. Soc., 97, 4176 (1975). 
The limitations of conventional spectroscopic studies are undoubtedly due to the low resolution which is inherent in straightforward absorption and emission experiments. This low resolution is a consequence of inhomogeneous broadening phenomena which obscure small spectroscopic splittings and which mask the electronic (homogeneous) line width of the chromophores in both the native and the denatured environment. One wellknown scheme for obtaining high optical resolution is the FLN technique (fluorescence line narrowing), where, via laser excitation, only specific sites within the inhomogeneous band are being excited and their narrowed fluorescence is being detected. ${ }^{10}$ This scheme, however, seems to break down for systems which exhibit efficient low-temperature photochemistry. ${ }^{11}$ It has, to our knowledge, not been applied to the study of native, light-harvesting pigments.

In this article we document that the photosynthetic pigments $C$-PC and allophycocyanin (APC), as well as synthetic dihydrobiliverdin (DHBV), which was used as a model system for the chromophore of $C$-PC and APC, undergo reversible low-temperature photochemistry. We utilize this newly discovered photochemical behavior to perform photochemical hole burning (PHB) experiments and thus achieve a more than 100 -fold increase in optical resolution. This enhanced optical resolution is used to distinguish between the chromophores in the different optical bands by studying their PHB behavior. It is also used to study the purely electronic (homogeneous) optical line width of the chromophore and to investigate the coupling strength between the electronic transitions and their protein environment. Finally, the PHB experiments shed light on the low-temperature reaction mechanism. Knowing the characteristic time constants of the photochemical reaction, we can also estimate the time constants for energy-transfer processes between chromophores because the latter processes are in competition with the observed low-temperature photochemistry.

\section{Experimental Section}

A. Sample Preparation. $C$-PC from the cyanobacterium Spirulina platensis was prepared as reported earlier. ${ }^{3}$ The method involves the breakage of the cells in a glass-ball mill, high-speed centrifugation of the crude extract to remove membrane-bound pigments, ammonium sulfate fractionation, and chromatography on DEAE-cellulose. On the basis of an analysis using polyacrylamide gel electrophoresis, our material contains $1.5 \%$ APC. The same amount has been estimated by comparing the relative area of the absorption bands in the low-temperature absorption spectrum (Figure 1) and by taking into account the known extinction coefficients of $C$.PC and APC. ${ }^{13}$ The samples, which were free of uncolored proteins, were dissolved in potassium phosphate buffer $\left(10^{-2} \mathrm{M}, \mathrm{pH} 7.5\right)$ and mixed with glycerol $(1: 3)$. The final solution had an optical density of 1.5 at $300 \mathrm{~K}$ in a $1-\mathrm{cm}$ cuvette.

2,3,7,8,12,13,17,18-Octaethyl-2,3-dihydro-1,19-(21 H,24H)-bilinedione was prepared according to the procedure of Cavaleiro and Smith ${ }^{14}$ and recrystallized from methanol. The sample was dissolved in a 3:1 ethanol/methanol mixture. The optical density of the sample at $300 \mathrm{~K}$ was the same as for C-PC.

B. Optical Experiments. The low-resolution ( $2.5 \AA)$ optical absorption spectra were recorded with a Cary 14 spectrophotometer and a lowtemperature helium-transfer cryostat. The higher resolution spectra were recorded with a $1-\mathrm{m}$ monochromator in second order with a dispersion of about $4 \AA / \mathrm{mm}$ and typical slit widths of $50-100 \mu \mathrm{m}$. The optical light path was such that the light of a 75-W xenon lamp was chopped at 90 $\mathrm{Hz}$ before being passed through the monochromator. The light from the exit slit was split by a 1:1 beam splitter. The deflected light beam was detected and amplified; it served as reference signal $I_{0}$. The undeflected light beam passed through the sample and was detected in a second red-sensitive photomultiplier. This signal $I_{2}$ was amplified with a second lock-in detector. Both $I_{0}$ and $I$ were processed in an analogue ratiometer and displayed on a strip-chart recorder.

(9) G. Wagnière and G. Blauer, J. Am. Chem. Soc., 98, 7806 (1976)

(10) R. I. Personov, E. I. Al'shits, and L. A. Bykovskaya, Opt. Commun., 6, $169(1972)$.

(11) J. Friedrich and D. Haarer, unpublished results.

(12) J. Friedrich, H. Scheer, B. Zickendraht-Wendelstadt, and D. Haarer, $J$. Chem. Phys., in press.

(13) W. Kufer and H. Scheer, Hoppe-Seyler's Z. Physiol. Chem., 360, 935 (1979)

(14) J. A. S. Cavaleiro and K. M. Smith, J. Chem. Soc., Perkin Trans. 1,2149 (1973)

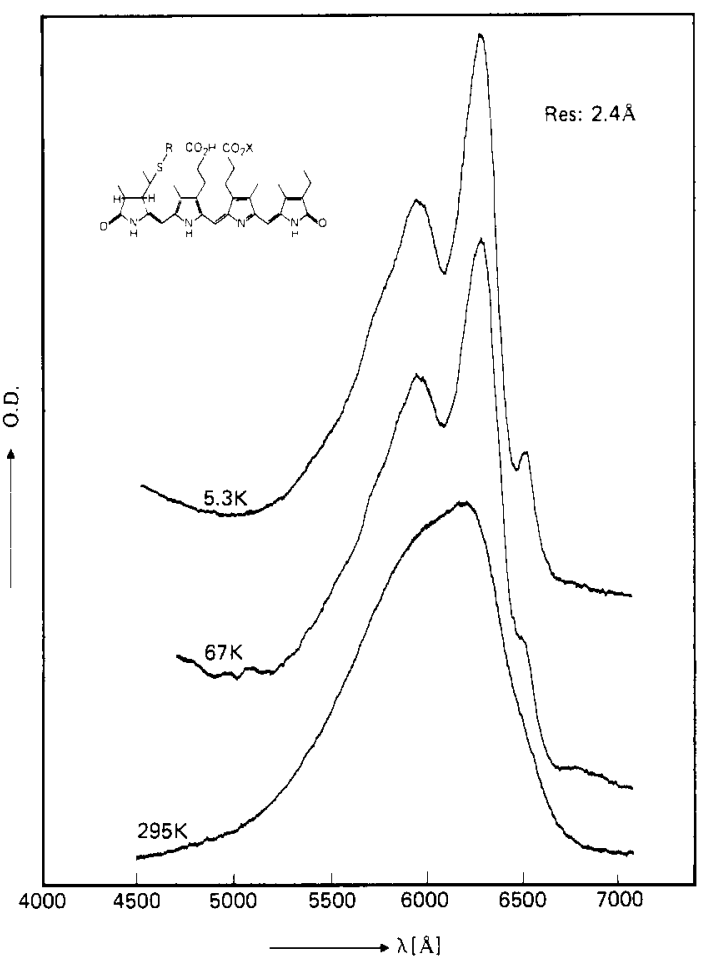

Figure 1. Visible absorption spectrum of $C$-phycocyanin as a function of temperature. Solvent: 3:1 glycerol/buffer solution. The lowest energy peak is due to allophycocyanin (1.2\%).

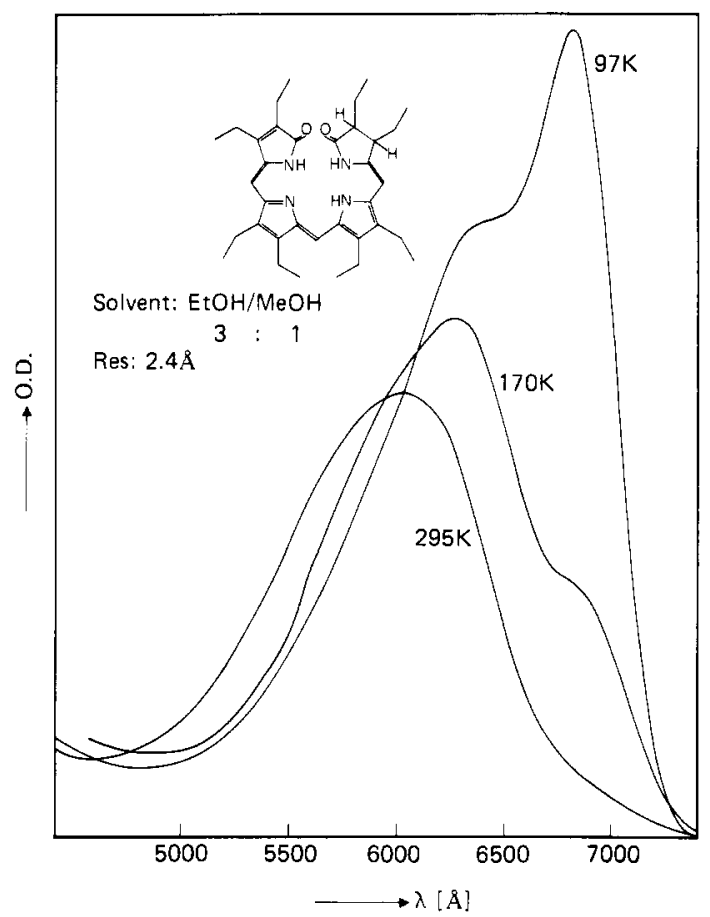

Figure 2. Visible absorption spectrum of the model chromophore of $C$-phycocyanin, DHBV, as a function of temperature.

The hole burning experiments were performed with a pulsed dye laser which had a bandwidth of about $0.02 \AA$. The pulse length was about 10 ns and the optical peak power of the light pulses was on the order of several tens of $\mathrm{kW}$ at pulse rates of $20-50 \mathrm{~Hz}$. In order to achieve uniform photochemistry, the light was not focused onto the sample but illuminated an area of several square millimeters. With the use of optical masks we made sure that the laser light path and the monochromator light path were identical. Typical hole burning times were on the order of seconds to minutes.

Results and Discussion

A. Low-Temperature Absorption Spectra of Native C-Phycocyanin, Allophycocyanin, and the Free Chromophore. Figure 1 
shows the temperature dependence of the lowest energy absorption band of native $C$-PC. The lower two traces, which were taken at room temperature and at $67 \mathrm{~K}$, agree with previously published data. ${ }^{5}$ The uppermost trace, which was taken at $5.3 \mathrm{~K}$, shows a further increase in resolution, which allows discrimination of three quite narrow absorption bands. The clearly discernible shoulder on the highest energy band $\left(\lambda_{\max } 5900 \AA\right)$ indicates that there are unresolved structures even at very low temperatures.

Figure 2 shows the temperature dependence of the lowest energy absorption band of DHBV, which is used as a model compound for the phycocyanobiline chromophore. The spectra were taken at three temperatures: 295,170 , and $97 \mathrm{~K}$. Below $97 \mathrm{~K}$ the spectrum does not exhibit measurable optical changes. A comparison between the native C-PC and the model chromophore can be summarized as follows:

Both absorption bands are, at $300 \mathrm{~K}$, similar in position and width, although of different oscillator strength. The native $C$-PC shows a slightly narrower width and indications of unresolved structure. Upon cooling, the center of gravity of the low-energy band of the native $C$-PC does not shift in position. Around $5 \mathrm{~K}$ the spectrum exhibits three well-resolved transitions with fairly narrow line widths. We attribute the two bands at 5900 and 6275 $\AA$ to $C$-PC and the band at $6500 \AA$ to APC. The model chromophore DHBV, however, shows a significant red shift, almost $2000 \mathrm{~cm}^{-1}$, and exhibits optical line widths which are at least a factor of 2 larger.

From these observations one can conclude that native $C$-PC has a smaller inhomogeneous line width than the free bile pigment. This smaller line width can be interpreted as being due to a well-defined conformation of the protein-chromophore assembly. ${ }^{5}$ The large shift of the band maximum of free DHBV and the increase in absorption and optical bandwidth is indicative of a change in protonation. ${ }^{5,15}$ It is interesting to note that the protein in native $C$-PC is obviously able to stabilize one degree of protonation, whereas the free chromophore undergoes a protontransfer reaction which seems to be reversible upon changing the sample temperature. As we show below, the specific state of protonation seems to be a crucial condition for the success or failure of photochemical hole burning.

B. Low-Resolution PHB Experiments. Photochemical hole burning is a phenomenon which occurs if one irradiates the optical absorption spectrum of a photolabile material with a narrow-band (laser) light source. ${ }^{16}$ The monochromatic irradiation initiates photochemistry of only those molecules which are in resonance with the laser frequency $\nu_{\mathrm{L}}$. The latter are selectivity "bleached" upon irradiation, and, hence, the absorption strength of the sample is reduced at the frequency $\nu_{L}$ and a "hole" appears in the spectrum. Molecules which have different optical transition energies due to slightly different solid-state environments do not absorb at the frequency $\nu_{\mathrm{L}}$ and, hence, are not affected by the narrow-band irradiation.

The detailed features of PHB spectra depend on the spread of molecular transition energies, the "inhomogeneous line width", which is a typical solid-state parameter. They also depend on the intrinsic width, the homogeneous line width, of the molecular transition involved. The latter needs to be narrow compared to the total inhomogeneous line width in order to facilitate the formation of a narrow photochemical hole. ${ }^{17}$

The requirement for a small homogeneous line width restricts PHB experiments to low temperatures $(2-20 \mathrm{~K})$, at which phonon scattering processes occur with low probability. The considered optical transition also needs to be free of broad and intense phonon sidebands (zero-phonon requirement). This latter requirement rules out many of the well-known photochromic materials which are often characterized by large Stokes shifts and broad multiphonon bands. A recent study of various possible PHB systems has shown the coexistence of zero-phonon lines and low-tem-

(15) S. E. Braslavsky, A. R. Holzwarth, E. Langer, H. Lehner, T. T. Mathews, and K. Schaffner, Liebigs Ann. Chem., in press.

(16) A. A. Gorokhovskii, R. K. Karlii, and L. A. Rebane, JETP Lett. (Engl. Transl.), 20, 216 (1974).

(17) D. M. Burland and D. Haarer, IBM J. Res. Dev., 23, 534 (1979).

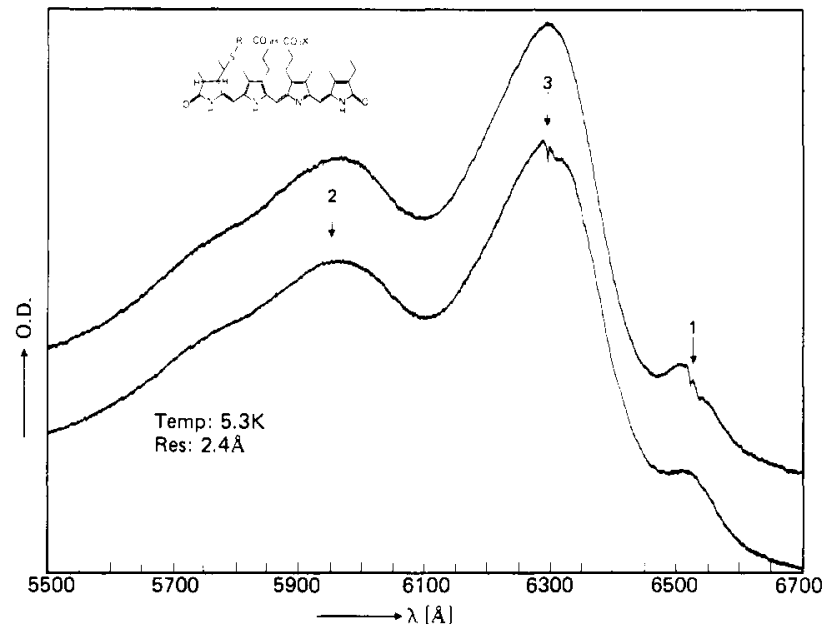

Figure 3. Low-resolution hole burning experiment in the visible spectrum of $C$-phycocyanin and allophycocyanin. Hole burning was performed sequentially as indicated by the numbers; i.e., the first irradiation occurred at $\nu_{1}$ (marked by 1 ) and the last at $\nu_{3}$ (marked by 3 ). It is important to note that the irradiation at $\nu_{2}$ refills the hole at $\nu_{1}$ (see text).

perature photochemistry is often not fulfilled. ${ }^{17,18}$

The three chromophores in native $C$-PC pigment are excellent examples for the above-mentioned PHB criteria. If we adopt the present interpretation of the native $C$-PC spectrum, then the two well-resolved bands at 5900 and $6275 \AA$ correspond to one and two chromophores, respectively. $3,5,19,20$ From the spectral data of the subunits, ${ }^{20}$ the single chromophore of the $\alpha$ subunit is tentatively assigned to the $5950-\AA$ band, whereas the two chromophores of the $\beta$ subunit are assigned to the $6275-\AA$ band. The less intense band around $6500 \AA$ corresponds to APC; here the two chromophores are unresolved even at very low temperatures. Figure 3 (top trace) shows the three long-wavelength bands at $5 \mathrm{~K}$ measured with low optical resolution $(2.5 \AA)$. The lower trace of Figure 3 shows the same spectrum after laser irradiation into the three bands at wavelength positions, which are marked with arrows. The two low-energy bands show a little negative "dip" in the absorption spectrum - a photochemical hole-whereas the high-energy band shows no photochemical hole.

In this section we will not elaborate on the spectral shape of the photochemical holes; we will only take the appearance of a hole as an indication of some kind of low-temperature photochemistry whose nature will be discussed in the following section. Thus we can conclude from Figure 3 that only one of the two low-lying bands of native $C$-PC and the APC band show lowtemperature photochemistry. The third, high-energy band obviously does not provide the appropriate structural or photochemical conditions.

If one performs the same PHB experiments, using the free DHBV pigment, as shown in Figure 4, one gets a similar result. The low-energy peak shows a narrow photochemical hole (see insert, Figure 4) whereas the broader high-energy peak does not exhibit a photochemical hole (insert). Since we know, that the long-wavelength peak is, most likely, a protonated form of the free biline chromophore, ${ }^{5,15}$ we can infer from the experiment that the state of protonation is crucial for the appearance of PHB phenomena. Similar results have been reported for smaller molecules (dihydroxyanthraquinone) where the deprotonated species does not exhibit hole burning, whereas the protonated species shows very high yield PHB. ${ }^{21}$

C. Narrow-Band PHB Experiments. In the previous section we dealt with the PHB experiments in a rather qualitative way, observing that certain bands did and others did not exhibit la-

(18) F. Graf, H.-K. Hong, A. Nazzal, and D. Haarer, Chem. Phys. Lett., 59, 217 (1978)

(19) R. E. Dale and F. W. J. Teale, Photochem. Photobiol., 12, 99 (1970).

(20) A. Binder, K. Wilson, and H. Zuber, FEBS Lett., 20, 111 (1972).

(21) F. Drissler, F. Graf, and D. Haarer, J. Chem. Phys. 72, 4996 (1980). 


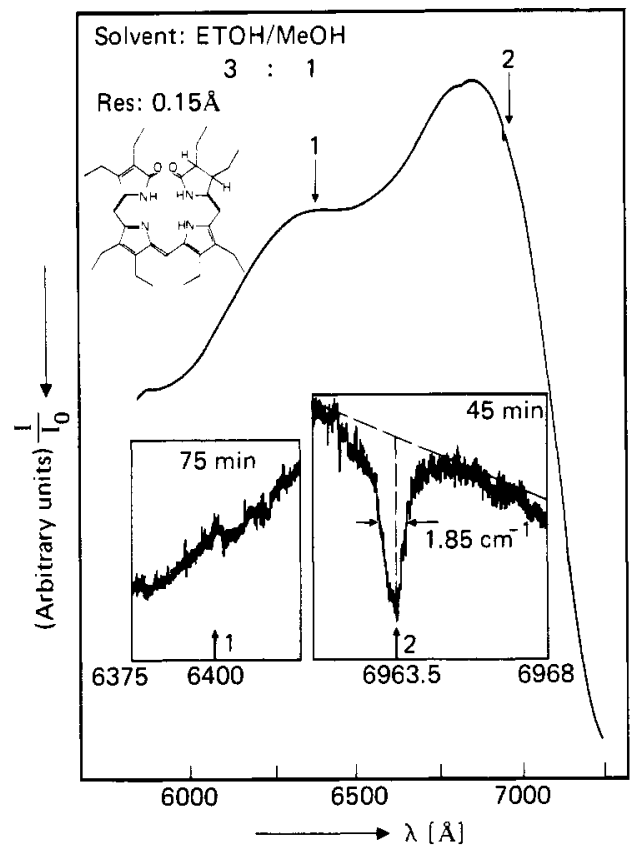

Figure 4. Low-temperature absorption $(1.8 \mathrm{~K})$ and hole burning experiments with the model chromophore of $C$-phycocyanin. Laser irradiation was performed at the positions 1 and 2 for 75 and $45 \mathrm{~min}$, respectively. The inserts represent expanded scans around these positions (see text).

ser-induced, narrow-band photochemistry. In this section we discuss the possible photochemical mechanism in more detail.

The $C$-PC chromophore is known to be photochemically very stable; otherwise it could not serve its role as a light-harvesting pigment, which has to absorb many photons and transfer the energy to the photoreactive center without decomposing or showing other irreversible side reactions or reactions which lead to radiationless deactivation (for instance, isomerizations ${ }^{22}$ ). In its denatured form, the $C$-PC chromophore has a cyclic, porphyrin-type conformation. Porphyrins are known to show PHB phenomena. ${ }^{23}$ In its native state it has an extended polyene-type conformation which is likely to exhibit external hydrogen bonding due to its $\mathrm{N}-\mathrm{H}$ and carbonyl groups. This latter conformation is likely to show light-induced proton rearrangements as have been discovered via PHB experiments. ${ }^{18}$ In the case of dihydroxyanthraquinone, for instance, ${ }^{18}$ it could be shown that the observed photochemistry is matrix dependent and does not occur in matrices which do not provide the possibility of external hydrogen bonding. Therefore, the observed photochemical holes were interpreted as being due to the breakage of an intramolecular hydrogen bond and the formation of an external intermolecular hydrogen bond. ${ }^{18,21}$ This model was consistent with the higher energy of the photoproduct $\left(1000 \mathrm{~cm}^{-1}\right)$, with the matrix dependence of the PHB mechanism, and with its $\mathrm{pH}$ dependence, i.e., its dependence on the state of protonation of the photoreactive species.

In the framework of this contribution we assume that a similar, light-induced proton rearrangement process occurs, and we try to test whether such an assumption is consistent with the experiments. Figure 5 shows a sequence of PHB experiments on the lowest photoreactive bands of $C$-PC and APC. Since this experiment was performed at $2 \mathrm{~K}$ and under high-resolution conditions, the holes are much more pronounced than in the previous figures. We believe that $C$-PC and APC are characterized by quite different molecular structures and hence the measured optical spectra can be considered as a superposition of two broad-band absorption curves. The argument of superposition also holds for the observed PHB features of the two involved

(22) H. Falk, N. Mueller, and T. Schlederer, Montash. Chem., 111, 159 (1980)

(23) S. Voelker, R. M. Macfarlane, H. P. Trommsdorf, and J. H. Van der Waals, J. Chem. Phys., 67, 1759 (1977).

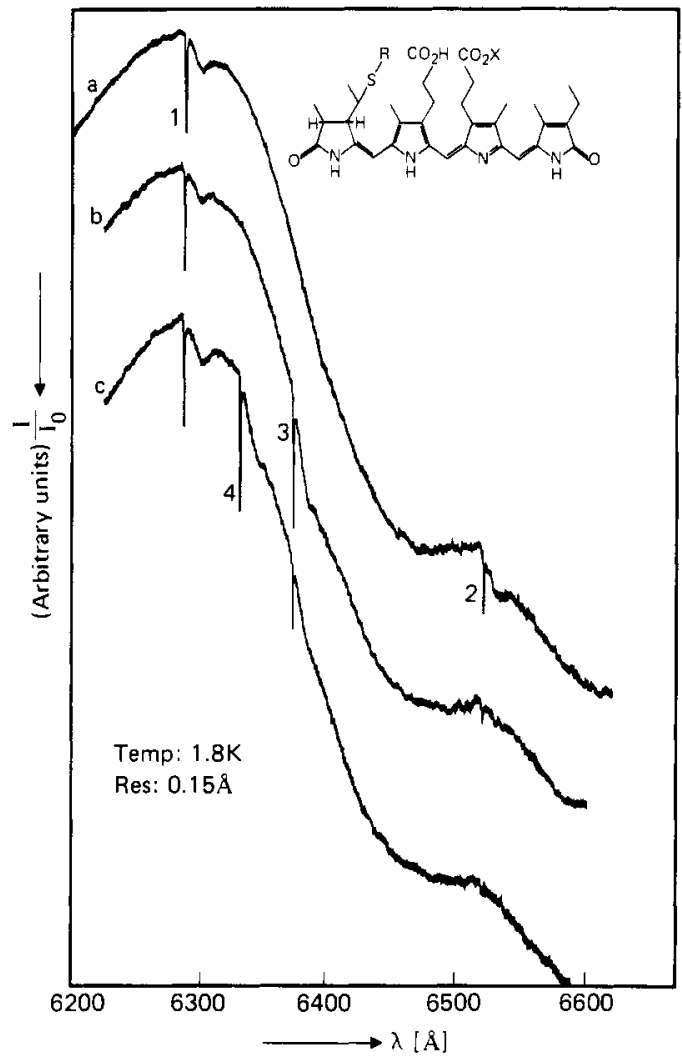

Figure 5. Multiple hole burning and filling experiments in the lowest bands of native $C$-phycocyanin and allophycocyanin $(1.8 \mathrm{~K})$. Again the hole burning irradiation was performed in the sequence as given by the numbers. (Hole burning at frequency $\nu_{3}$ refills the hole at $\nu_{2}$ (see text).)

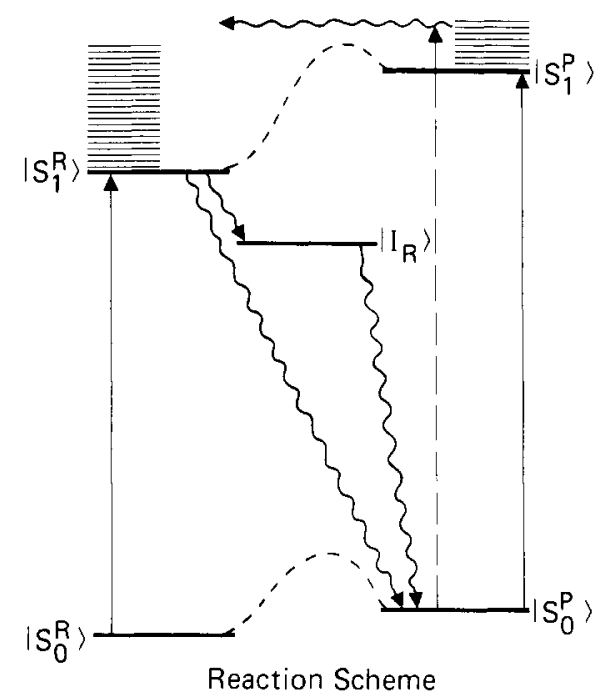

Figure 6. Level and reaction scheme for the hole burning photochemistry of $C$-phycocyanin. The straight lines correspond to optical transitions into the electronic origins (solid lines) and into phonon states (broken line), respectively. The wavy lines correspond to the various radiationless pathways. The narrowly spaced states above the excited-state levels correspond to phonon states. The superscripts $\mathrm{R}$ and $\mathrm{P}$ refer to the reactant and the photoproduct states, respectively. The dotted lines symbolize activation barriers in the ground and excited states, respectively.

species. Laser irradiation at frequencies $\nu_{1}$ and $\nu_{2}$ leads to narrow photochemical holes whose line shapes will be discussed in the next section. Laser irradiation at the intermediate frequency $\nu_{3}$ (trace b), which is well outside the envelope of the lowest energy band (APC), refills the hole at frequency $\nu_{2}$ and creates a third hole. It should be noted that there is absolutely no change in the hole depth at frequency $\nu_{1}$. Similarily, irradiation at frequency 


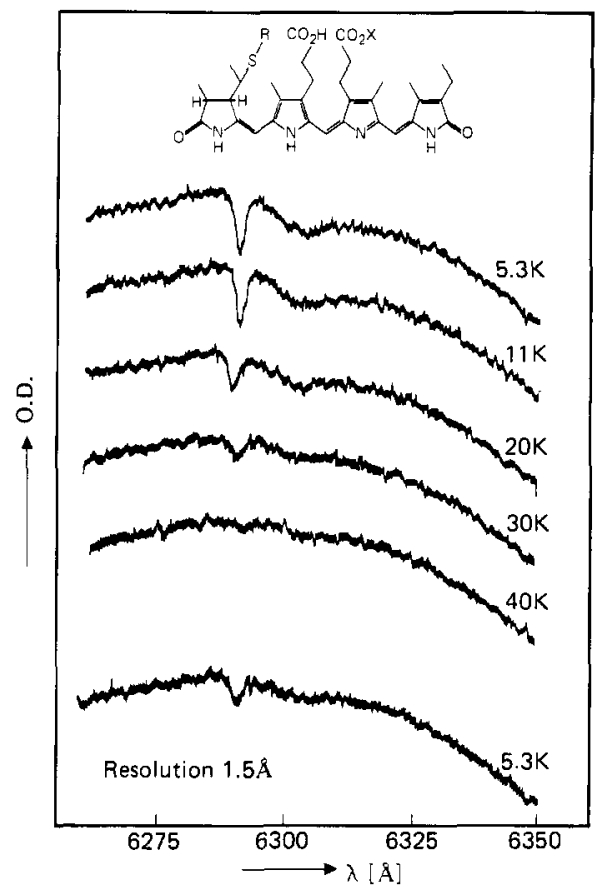

Figure 7. Temperature dependence of a photochemical hole in native $C$-phycocyanin. The hole was burnt at $5.3 \mathrm{~K}$. The lowest trace represents a hole spectrum after the thermal cycling process.

$\nu_{4}$ eliminates part of the hole at $\nu_{3}$ and leaves $\nu_{1}$ unchanged (trace c).

The above results can be understood readily if one assumes a photoreactive scheme in which the photoproduct absorbs at higher energies (higher with respect to the position of the hole) and in which light-induced reverse photochemistry occurs (Figure 6). This would be analogous to experiments on smaller, hydrogenbonded molecules. ${ }^{18,21}$ The remarkable feature of the observed reverse photochemistry is the fact that a hole (for instance, at frequency $\nu_{2}$ ) can be filled by narrow-band laser irradiation into an arbitrary, higher lying state (for instance, at $\nu_{3}$ ). This surprising result requires, in our opinion, the notion of a very broad (phonon broadened) photoproduct absorption. Figure 7 shows a photochemical hole as a function of temperature in a low-resolution experiment. The hole broadens and disappears at about $40 \mathrm{~K}$. Only part of the hole can be recovered if one recycles the system back to low temperatures. More than half of the hole has been refilled thermally during the heating cycle. From these experiments and from the fact that, at room temperature, there is no observable net photochemistry, we can draw some interesting conclusions (Figure 6): First, from the thermal behavior of the hole burning photochemistry we infer that the ground state of the photoproduct is above the ground state of the reactant. Second, from the observation that hole burning at the laser frequency $\nu_{\mathrm{L}}$ fills holes at lower frequencies $\left(\nu_{\mathrm{L}}-\Delta\right)$ but does not affect holes at higher frequencies $\left(\nu_{L}+\Delta\right)$ we infer that the photoproduct absorbs at shorter wavelengths than the reactant. Knowing the relative energies of the ground states, we can safely conclude that the first excited state of the product is above the first excited state of the reactant and, hence, is not accessible in a reaction which involves the two lowest excited singlet states (see Figure 6). Therefore, the first step in the photochemical reaction is radiationless relaxation to a lower state, most probably the triplet state, from which the proton rearrangement process occurs. (The triplet state of a free biliveredin has recently been observed. ${ }^{24}$ ) These conclusions link the time scale of the photoreaction to the intersystem crossing process.

It should be noted that the above $\mathrm{PHB}$ experiments do have quite a few features in common with photophysical hole burning experiments. ${ }^{25,26}$ However, we believe that the position of the

(24) E. J. Land, Photochem. Photobiol., 29, 483 (1979).

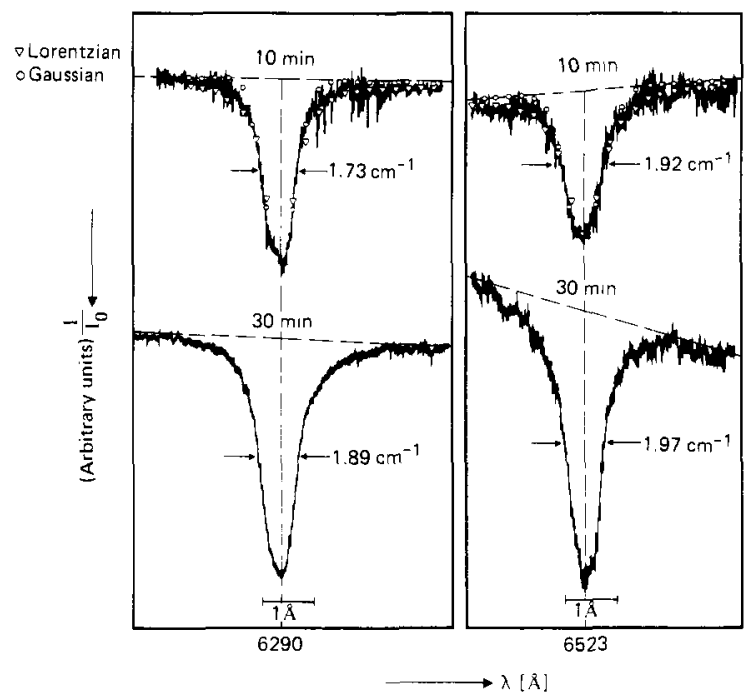

Figure 8. Expanded, high-resolution scans of holes 1 and 2 (see Figure 3) for two different burning times. Fits to Lorentzian or Gaussian line shapes are marked by triangles and circles. ${ }^{28}$

photoproduct, the sensitivity towards protonation of the chromophore, and the selectivity with respect to the three different low-energy bands are strong arguments in favor of a direct involvement of molecular hydrogen bonds in the observed hole burning experiments. In the following section of this paper, we discuss the detailed line-shape features of the observed photochemical holes. This part will provide most of the spectroscopic and mechanistic insights into details of the light-harvesting process.

D. Shape and Width of the Photochemical Holes. 1. ZeroPhonon Criterion. It is important for a detailed understanding of the role of the protein in stabilizing the chromophore whether the latter is strongly attached to its protein environment or whether it forms a loose and floppy configuration which is likely to show molecular distortions in the excited state of the light-harvesting chromophore. The narrowness of the photochemical holes (Figure 8 ) and the small intensity of the corresponding phonon wings in Figure 5 reflect a rigid attachment of the bile pigment to its protein environment. The phonon wing, which is a measure of the chromophore coupling to its environment, is the broad satellite which accompanies each hole at the low-energy side of the spectrum (see Figure 5). It was shown that, for short burning times, its intensity is a direct measure of the electron-lattice coupling strength which can be evaluated quantitatively. ${ }^{27}$ From a comparison with smaller molecules (dihydroxyanthraquinone ${ }^{27}$ ) we can estimate that the chromophore is in the weak phonon coupling limit. This is an important observation because it means that only very little of the absorbed photon energy is transformed into heat by phonon relaxation. Instead, most of the energy is available for energy transfer to the photoreactive center.

2. Width and Line Shape of the Zero-Phonon Holes. Figure 8 shows expanded spectra of photochemical holes in the lowest band of native $C$-PC $(6290 \AA)$ and APC $(6523 \AA)$. The hole widths are less than $2 \mathrm{~cm}^{-1}$ and can be well described by a Lorentzian line shape. ${ }^{28}$ The extrapolated hole widths for zero burning time are 1.7 and $1.9 \mathrm{~cm}^{-1}$ for the higher and lower energy bands, respectively. If one interprets the hole width as being due to a dynamic process in the photosynthetic pigment, then it would reflect a typical relaxation time on the order of several picoseconds. A better understanding of this dynamical process would require more microscopic knowledge about the light-harvesting center than

(25) J. M. Hayes and G. J. Small, Chem. Phys., 57, 151 (1978)

(26) J. M. Hayes and G. J. Small, Chem. Phys. Lett., 54, 435 (1978).

(27) J. Friedrich, J. D. Swalen, and D. Haarer, J. Chem. Phys., 73, 705 (1980)

(28) The small differences between a Gaussian and a Lorentzian fit only show up in the outer wings of the curves and therefore it does not matter (for our present level of data accuracy) that we have plotted $I_{0} / I$ rather than log $\left(I_{0} / I\right)$. 


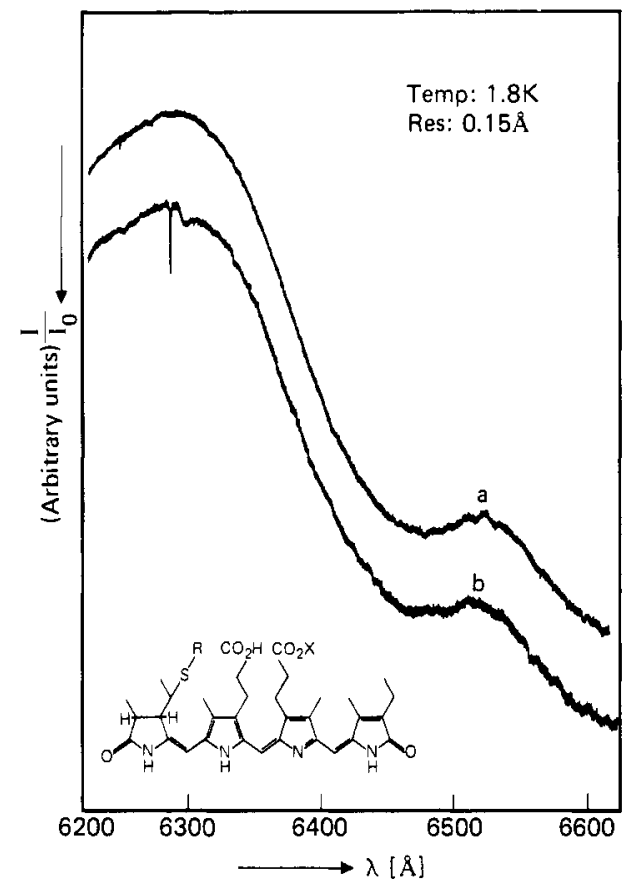

Figure 9. Hole burning in the lowest energy band of $C$-phyocyanin: trace a, before irradiation; trace b, after irradiation at 6290 (10 min). Hole burning into the $C$-PC species does not affect the lower energy APC band.

is presently available. There are, however, several important conclusions which can be drawn from the above experiments.

Since a photochemical hole burnt at $6290 \AA$ appears in neither the lower $(6523 \AA)$ nor the higher $(5950 \AA)$ energy band, we can safely conclude that these bands represent three different species. The lowest energy band has already been assigned as being due to APC. Figure 9 shows clearly that hole burning into the $C$-PC band at $6290 \AA$ does not affect the APC band at $6523 \AA$. The fact that the band at $5940 \AA$ does not show any hole burning could be due either to a local conformation which does not undergo light-induced proton rearrangement or to a very fast excitation transfer which is not energy selective and hence broadens the hole to the extent that it does not appear in the spectrum. ${ }^{12}$ At the present stage of our investigation, we cannot distinguish between the two possibilities.

Another, quite unexpected result of the above hole burning experiments, which is in contrast to results on other antenna pigments, ${ }^{12}$ is the fact that none of the holes exhibits "satellite holes" of measurable intensity. Such multiple holes could be expected in spectral ranges where the absorption of various chromophores overlaps and where energy transfer can channel the photon energy into well-defined "trap states" of lower energy. It is believed, for instance, that the $C$-PC antenna contains, in analogy with phycoerythrin, ${ }^{29}$ three chromophores. Two of those chromophores give rise to well-resolved optical bands; the absorption of the third chromophore, however, is not known. It is believed that the latter chromophore absorbs in the band which appears at the low-energy side of the spectrum $(6290 \AA)^{5,20}$ and hence this absorption peak is a superposition of two inhomogeneously broadened optical lines. The fact that only one, comparatively narrow hole is measured in this lowest energy absorption band either can be interpreted as a lack of fast energy transfer between various chromophores at rates comparable to the photochemical reaction rates (ns) or can be taken as evidence that the absorption of the third chromophore does not coincide with the lowest absorption peak. Recent experiments on energy transfer in $C$-PC from a different organism ${ }^{30}$ have yielded transfer times in the range of $50 \mathrm{ps}$. However, these experiments were done at room temperature where the rates for nonresonant energy transfer may be different by more than an order of magnitude.

\section{Summary}

We have utilized the novel spectroscopic method of photochemical hole burning to gain insight into the functioning of the native pigments of $C$-phycocyanin. The 100 -fold increase in optical resolution over conventional spectroscopic methods has led to a better understanding of the nature of the electronic transitions of the bile pigments and has brought us to the conclusion that the chromophores are quite rigidly attached to the surrounding protein (zero-phonon line criterion). We have reestablished the notion that the various bands can be considered as independent electronic origins with different photochemical behavior. Last but not least, we have shown that there is a low-yield photochemical reaction mechanism, on which the PHB chemistry is based, which converts a small percentage of the incident photons into the radiationless energy which is dissipated during the photochemical cycle. Therefore, at low temperatures, the total efficiency of the light-harvesting unit is bound to be somewhat smaller than 1 , in agreement with a measured fluorescence quantum yield of about 0.8 for isolated $C$-PC and APC. ${ }^{31}$ Finally, it should be noted that photochemistry is the major pathway of deexcitation in phytochrome and phycochrome sensory pigments of green plants and cyanobacteria. ${ }^{32}$ These pigments contain chromophores very similar to those of $C$-PC and PAC, and there is recent evidence that both pigments become reversibly photochromic upon partial denaturation of the protein. ${ }^{33}$

Acknowledgment. This study was supported by the Office of Naval Research (D.H. and J.F.) and by the Deutsche Forschungsgemeinschaft.

(29) B. Zickendraht-Wendelstadt, J. Friedrich, and W. Ruediger, Photochem. Photobiol., 31, 367 (1980).

(30) T. Kobayashi, E. D. Degenkolb, R. Bersohn, P. M. Rentzepis, R. MacColl, and D. S. Berns, Biochemistry, 18, 5073 (1979).

(31) J. Grabowski and E. Gantt, Photochem. Photobiol., 28, 39 (1978).

(32) K. Ohki and Y. Fujita, Plant Cell. Physiol, 20, 483 (1979).

(33) T. Ohad, R. K. Clayton, and L. Bogorad, Proc. Natl. Acad. Sci. U.S.A., 76, 5655 (1979). 